\title{
Une formation pour développer le Leadership des médecins cadres
}

\author{
Pour répondre aux nouveaux besoins de compétences de management des mé- \\ decins cadres, une formation spécifique pour les médecins a été développée en \\ mettant l'accent sur les outils indispensables de management de proximité mais \\ également sur la possibilité de partager les expériences du quotidien.
}

\section{Bernard Vermeulen, \\ Pierrette Chenevard ${ }^{b}$ \\ a Directeur médical hôpital fribourgeois \\ b Directrice générale Espace compétences SA}

\begin{abstract}
Introduction
En 2004 l'Académie Suisse des Sciences Médicales (ASSM) publiait une brochure intitulée «buts et missions de la médecine au début du $21^{\text {ème }}$ siècle». Elle identifie les défis annoncés à court et moyen termes de l'organisation et de la gestion des processus de santé. Dans l'avant-propos, le Prof. Dr Dieter Bürgin écrit: «la médecine se trouve au cœur de cette crise liée à la transformation de notre société» [1]. Dans le chapitre $\mathrm{V}$ «les missions de la médecine et leur réalisation», diverses missions y sont développées notamment celles qui mettent l'accent sur les compétences globales des médecins y compris la gestion de l'institution qu'ils dirigent ou qui les emploie: «dans toutes les professions médicales, il faut veiller à ce que la relève soit assurée et à ce que des personnes ayant le profil soient motivées à exercer ces professions.»
\end{abstract}

\section{«Avec l'administration de l'hôpital, depuis toujours, les médecins entretiennent une relation amour-haine.»}

1 Buts et missions de la médecine au début du XXI ${ }^{\text {ème }}$ siècle: Académie Suisse des Sciences médicales: 2004.

2 Debrosse D. La nouvelle gouvernance hospitalière. adsp (actualité et dossier en santé publique). 72 , septembre 2010.

\section{Correspondance:}

Prof. Dr Bernard Vermeulen

Directeur médical

hôpital fribourgeois

Chemin du Pensionnat

CH-1708 Fribourg

Tél. 0264268839

bernard.vermeulen[at]h-fr.ch
Les compétences professionnelles attendues incluent donc une compétence managériale. Celle-ci peut être déclinée différemment suivant la taille et les besoins de l'infrastructure médicale dans laquelle le médecin exerce son activité. Les médecins-chefs et médecins cadres des hôpitaux publics ou privés doivent gérer leur service composé de médecins, de paramédicaux et autres personnels. Avec l'administration de l'hôpital, depuis toujours, les médecins entretiennent une relation amour-haine. Ils sont toutefois des partenaires indissociables pour résoudre ensemble les défis médico-économiques imposés par la société. Les composants de la mission d'un hôpital d'aujourd'hui sont la qualité et la sécurité des soins aux patients, en respectant l'efficience pour garantir la couverture et/ou la rentabilité. Le terme de nouvelle gouvernance hospitalière caractérise cette collaboration. Elle responsabilise le personnel soignant et non soignant, élargit l'autonomie de l'établissement et instaure des contrats entre les dif-

\section{Eine Ausbildung zur Förderung des Leaderships Leitender Ärzte}

Die Spitalorganisation und das Spitalmanagement befinden sich in stetem Wandel. Kürzlich wurde der Begriff «Neue Spitalführung» (Nouvelle gouvernance hospitalière) eingeführt. Diese zeichnet sich durch eine faktische Zusammenarbeit zwischen der Verwaltung, den Kaderärzten und dem Pflegepersonal aus und zieht das Personal in die Verantwortung. Sie erweitert die Autonomie der Institutionen und führt Leistungsverträge zwischen den verschiedenen Akteuren des Gesundheitswesens ein. Um auf die neuen Bedürfnisse bei Managementkompetenz von Kaderärzten einzugehen, wurde eine spezifische Ausbildung für Ärzte entwickelt. Sie legt das Hauptaugenmerk auf die unverzichtbaren Basisinstrumente der Führung, aber auch auf einen Erfahrungsaustausch über den Alltag. Das Ausbildungskonzept, die identifizierten Ziele und eine Evaluierung durch die Teilnehmenden werden diskutiert.

férents acteurs de la santé. Pour les médecins cadres, le leadership médical s'en trouve profondément modifié [2]. Mais contrairement aux cadres administratifs des hôpitaux, les médecins n'ont que peu ou pas de connaissances des concepts de management, ce qui peut perturber les collaborations au sein d'une institution.

Avec l'introduction en Suisse du nouveau financement hospitalier par DRG (Diagnosis related groups) en 2012, une gestion plus efficiente des ressources à disposition est devenue impérative pour assurer la survie de l'hôpital public. Pour les médecins cadres il devient impératif de se former aux exigences de la nouvelle gouvernance. Dans les pays ayant introduit 


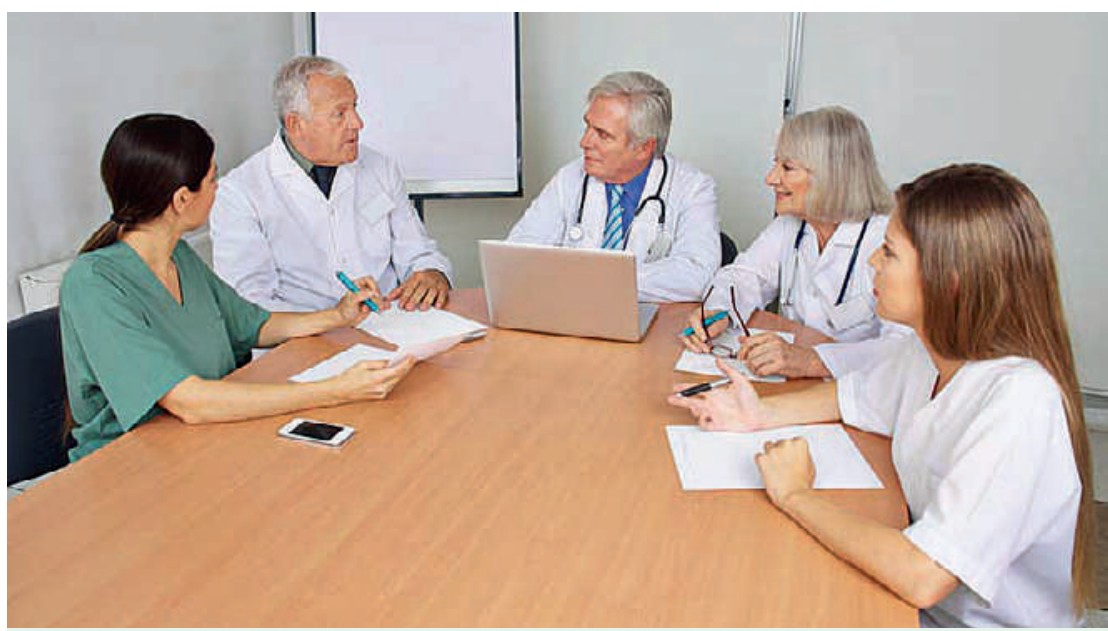

Un enseignement basé sur la dynamique de groupe restreint favorisant la création de liens de confiance et d'échanges autour de thèmes du vécu professionnel peut pédagogiquement se montrer bénéfique pour tous.

les DRG dès les années 1990, la littérature consacrée à la gestion hospitalière manifeste d'une augmentation des demandes de formation de type managérial pour les médecins cadres. Par exemple en 2006, le centre de recherche en Sciences de Gestion de SaintEtienne a organisé [3] un colloque intitulé «Trajectoire d'appropriation des principes de nouvelle gouvernance hospitalière par les médecins». En 2010, l'Harvard Business Review sous la plume de Thomas H. Lee publie un Spotlight «Turning Doctors into Leaders» [4]. Des formations pour permettre aux médecins cadres d'acquérir des compétences managériales ont vu le jour dans tous les pays. Les objectifs et les méthodologies pédagogiques utilisés pour ces formations sont très différents suivant le niveau de validation à atteindre. Certaines ont fait recours aux nouvelles méthodes pédagogiques comme la dynamique des groupes restreints, d'autres sont restées plus classiques en dispensant des cours de type excathedra. La dynamique de groupe restreint permet une acquisition des connaissances et des compétences par échange d'expériences autour de thèmes prédéfinis [5]. Elle permet de découvrir l'importance de la mise en commun des compétences de chacun pour atteindre une meilleure compétence collective (team working), maillon clé de la gestion des équipes de soins et réussite de projets hospitaliers [6].

Après plusieurs années d'activité comme médecin-chef d'un service d'urgence puis directeur médical d'un réseau hospitalier, le développement d'une formation orientée plus spécifiquement vers les besoins de mes collègues m'est apparu comme nécessaire. Ceux-ci ont régulièrement exprimé leur souhait, non pas de devenir des «top managers» mais de disposer d'outils adaptés pour comprendre les processus de la gouvernance hospitalière et pouvoir y participer plus activement. Les institutions conscientes de la nécessité d'introduire un concept de nouvelle gouvernance hospitalière ont plusieurs difficultés à résoudre: identifier ou développer une forma-
7 Espace compétences: Cully. http://espace-competences.ch tion adaptée à l'institution et entre autres aux médecins? Comment motiver les médecins cadres? Pour les médecins cadres intéressés: comment libérer du temps? Comment faire valider auprès des sociétés savantes de tutelle cette formation dans un parcours professionnel comme formation continue?

Une formation se doit d'énoncer des objectifs précis et compréhensibles pour que la valeur ajoutée soit rapidement identifiée au quotidien. Cette formation se doit parallèlement d'être un moment de «respiration» dans la lourde charge des activités quotidiennes des médecins cadres. Ils doivent aussi y trouver du plaisir. L'accumulation d'expériences professionnelles complexes peut être émotionnellement lourde à porter et causer des épuisements, souvent reconnus trop tardivement, voire méconnus. Un enseignement basé sur la dynamique de groupe restreint favorisant la création de liens de confiance et d'échanges autour de thèmes du vécu professionnel peut pédagogiquement se montrer bénéfique pour tous.

En nous adressant au centre de formation Espace compétences, Lavaux (VD), au centre de formation reconnu par $\mathrm{H}+$ et certifié Eduqua, nous réunissions un environnement pédagogique structuré, habitué à développer des formations pour les professionnels de la santé, un lieu convivial et de recourir à des intervenants très au fait des réalités du monde de la santé [7]. La direction d'Espace compétences a immédiatement manifesté son intérêt.

\section{Identification des objectifs d'apprentissage organisation et évaluation}

La formation management pour médecins cadres couvre six thèmes: conduite du changement, gestion de projets, communication et gestion de conflits, gouvernance d'une structure hospitalière, risk-management, gestion du stress et épuisement professionnel. Pour chaque thème les compétences générales attendues ont été définies par des objectifs spécifiques centrés sur le besoin des médecins cadres.

Dans le tableau 1 sont décrits les objectifs d'enseignement du premier module intitulé «Stratégie du changement». Pour chaque module, un sous-objectif a été identifié pour favoriser un temps de réflexion plus humaniste et/ou philosophique des réalités sociétales d'aujourd'hui. Dans un processus de changement, le rôle du leader est primordial. Il a été fait appel à un professeur de philosophie pour développer avec les participants une réflexion, au travers d'écrits philosophiques anciens et récents, de l'importance et de l'image que véhicule le leader de par son comportement.

La complexité de chaque sujet et les objectifs identifiés exigent pour être traités correctement au moins 32 heures d'enseignement. Ils sont répartis sur 2 périodes de 2 jours séparés d'au moins 4 semaines afin de laisser du temps pour les lectures proposées lors de la première session. Des recommandations ont été faites aux enseignants afin de favoriser 
Tableau 1

Module 1: Conduite du changement.

$\begin{array}{llc}\text { Axe de compétences } & \text { Compétences générales } & \text { Compétences spécifiques } \\ \text { Compétences } & \text { Conduite du changement } & - \text { Analyser le contexte du projet de } \\ \text { managériales } & \text { Accompagner les } & \text { changement } \\ & \text { évolutions stratégiques de } & - \text { Connaître les diverses représentations du } \\ \text { l'institution en travaillant la } & \text { changement } \\ \text { planification, l'organisation, } & - \text { Connaître et Identifier les phases du } \\ \text { le pilotage et l'intégration } & \text { processus du changement } \\ \text { d'un projet } & - \text { Evaluer les avantages et les inconvénients } \\ & \text { du changement } \\ & - \text { Maîtriser la dimension humaine d'un } \\ & \text { projet de changement } \\ & - \text { Anticiper les résistances et les blocages } \\ & \text { possibles } \\ & - \text { Négocier et se mettre d'accord sur le } \\ & \text { processus du changement } \\ & - \text { Mettre en œuvre et évaluer les change- } \\ & \text { ments }\end{array}$

Thèmes / Contenus

$\rightarrow$ Le rôle du médecin-cadre et ses leviers d'action dans le processus changement

$\rightarrow$ Méthodes et outils de conduites du changement adaptés à la logique et au degré de changement recherché

$\rightarrow$ Cycle du changement

$\rightarrow$ Leviers du changement

$\rightarrow$ Typologie des causes de résistances

$\rightarrow$ Mécanismes à l'origine des comportements humains face aux changements

$\rightarrow$ Types de pouvoir, enjeux de pouvoir

$\rightarrow$ Méthodes et outils de négociation

$\rightarrow$ Méthodes d'analyse, de travail et de prise de décision pour une gestion satisfaisante des changements (Decision Making)

des ateliers en petits groupes suivis de restitutions structurées. Trois modules sont enseignés la $1^{\text {ère }}$ année, trois modules l'année suivante.

La validation de la formation a été énoncée clairement sur le fond et la forme de manière à gagner la confiance des participants et des employeurs qui financent la formation. Les objectifs attendus sont:

- mettre en lien les apports théoriques et leurs transferts concrets sur le terrain,

- mettre en perspective les apports théoriques,

- prendre conscience du processus d'apprentissage,

- développer une posture réflexive par rapport à la position de cadre dans l'institution.

Ces 4 points ont été évalués chez les participants de la $1^{\text {ère }}$ volée qui ont suivi la formation entre octobre 2011 et juin 2013. Les avis des apprenants ont été recueillis sur le contenu et la forme de chaque module. A l'issue de la première année (juin 2012), chaque participant est entendu lors d'un entretien structuré de 45 minutes sur un sujet préparé à l'avance et évalué par deux enseignants selon une grille de lecture structurée des connaissances attendues. A la fin de la deuxième année, un travail rédactionnel sur la base Artikel d'une synthèse des réflexions personnelles menées durant le cursus de la formation est rendu et évalué par les enseignants (juin 2013).

Une demande de reconnaissance sous la forme Wollen Sie diesen Artike kommentieren? Nutzen Sie dafür die Kommentarfunktion in der OnlineVersion oder sehen Sie nach, was Ihre Kolleginnen und Kollegen bereits geschrieben haben: www.saez.ch/ aktuelle-ausgabe/ interaktive-beitraege/ de crédit de formation continue FMH a été introduite auprès de l'ISFM/FMH. Sur la base des documents fournis, l'ISFM/FMH a accordé 50 crédits de formation continue par année de formation.

\section{Conclusion: Evaluation de la formation par les participants}

Lors de la remise des certificats aux participants de la première volée, nous leur avons demandé d'ex- primer leur vécu sur les 2 ans qu'a durée cette formation. En guise de conclusion, je leur donne la parole.

B.C., médecin adjoint spécialisée en médecine palliative: «Un médecin qui assume des responsabilités est inévitablement amené à gérer d'autres aspects de sa fonction de clinicien, à savoir la conduite du changement, la gestion de projets, les conflits de personnes ou d'équipes, les conflits avec le monde de l'administration, le leadership, la gestion du stress, etc. Grâce à cette formation, mêlant connaissances et pratique, et grâce au fait qu'elle permet d'échanger avec d'autres médecins venant de différentes spécialités, j'ai pu acquérir une autre vision de la gestion et surtout acquérir de nombreux outils de travail.»

O. C., médecin chef en Gynécologie Obstétrique: «Il s'agit de cours passionnants où se mélangent enseignement et exercices pratiques qui nous permettent de traverser notre quotidien avec un meilleur recul. Lorsque votre position hiérarchique évolue, la possibilité de partager avec vos confrères certaines situations, de les aborder avec des professionnels qui ont une habitude et une expérience évidente, même en dehors du côté médical - par exemple avec un commandant de bord de l'aviation - est un atout supplémentaire indéniable.»

K. J-V., cheffe de clinique en médecine gériatrique et réadaptation: «De nos jours il est indispensable pour les médecins cadres d'avoir des connaissances managériales afin de pouvoir au mieux s'intégrer dans le milieu hospitalier qui est en constante évolution. Ce cours est absolument idéal pour l'acquisition de ces connaissances. Elles nous sont délivrées d'une manière précise et intéressante tout en maintenant une ambiance conviviale.» 